\title{
A Bridge Not Too Far: Linking Disciplines Through Molecular Imaging Probes
}

\author{
John F. Valliant \\ Departments of Chemistry and Medical Physics and Centre for Probe Development and Commercialization, McMaster University, \\ Hamilton, Ontario, Canada
}

CE credit: For CE credit, you can access the test for this article, as well as additional JNMT CE tests, online at https://www.snmmilearningcenter.org. Complete the test online no later than September 2016. Your online test will be scored immediately. You may make 3 attempts to pass the test and must answer $80 \%$ of the questions correctly to receive $1.0 \mathrm{CEH}$ (Continuing Education Hour) credit. SNMMI members will have their CEH credit added to their VOICE transcript automatically; nonmembers will be able to print out a CE certificate upon successfully completing the test. The online test is free to SNMMI members; nonmembers must pay $\$ 15.00$ by credit card when logging onto the website to take the test.

The field of nuclear medicine will rely increasingly on the discovery, proper evaluation, and clinical use of molecular imaging probes and on collaborations. Collaborations will include new initiatives among experts already involved in the field and with researchers, technologists, and clinicians from different areas of science and medicine. This article serves to highlight some of the opportunities in which molecular imaging and nuclear medicine in conjunction with probe development, new imaging technologies, and multidisciplinary collaborations can have a significant impact on health care and basic science from the perspective of a person involved in probe development. The article emphasizes breast cancer, but the concepts are readily applied to other areas of medicine and medical research.

Key Words: probes; new technologies; breast cancer; collaboration

J Nucl Med Technol 2016; 44:173-183

DOI: 10.2967/jnumed.109.068312

\section{$\mathbf{N}$} uclear medicine is a unique field in that it relies on habitual collaboration between groups possessing disparate expertise. Nuclear medicine physicians work collaboratively on a daily basis with imaging physicists and nuclear medicine technologists who directly and indirectly rely on partnerships with biologists, chemists, engineers, and medical research teams to develop, validate, and produce radiopharmaceuticals. The future of nuclear medicine involves exploiting the full potential of molecular imaging and targeted radiotherapy and will require building on the existing culture of collaboration. To be truly successful, nuclear medicine

\footnotetext{
Received Dec. 14, 2009; revision accepted Apr. 1, 2010.

For correspondence or reprints contact: John F. Valliant, 1280 Main St. W., Hamilton, ON L8S 4K1, Canada.

E-mail: valliant@imagingprobes.ca

COPYRIGHT (c) 2016 by the Society of Nuclear Medicine and Molecular Imaging, Inc.
}

will need to form bridges to groups outside the present sphere of collaboration-the underlying theme of this article.

Society is facing challenging times that will shape the future of probe development. There has been a significant downturn in the world's economy that is affecting economic decisions made by consumers and governments, particularly with respect to health care. The world is in the midst of a protracted and ongoing isotope shortage, both an acute shortage associated with the shutdown of the reactor at Chalk River, limiting the supply of ${ }^{99} \mathrm{Mo}$ and ${ }^{99 \mathrm{~m}} \mathrm{Tc}$, and a chronic underfunding of cyclotron laboratories. Economic pressures are affecting the way decisions are made about which new diagnostic technologies will ultimately be funded. This effect is true in public, private, and hybrid-model health-care systems, in which groups developing new probes must be conscious about both the health and the economic impacts new agents will have. There is also a tightening of regulatory requirements around the security and transportation of nuclear materials, the production of conventional radiopharmaceuticals, and the translation of novel agents from the laboratory to the clinic.

Throughout history, challenging times have provided the motivation needed to bring about paradigm shifts and transformative innovations. Soon, for instance, we can expect to see changes brought about by the isotope shortage, which will make the field rethink the mix of isotopes and agents that are used in routine clinical practice. Economic forces will drive technologic developments that enable earlier detection and more accurate staging of disease, a more effective and expedient choice of therapeutic intervention, and greater use of outpatient procedures.

To begin responding to today's challenges, probe development must move beyond early detection and staging and look for new opportunities to positively affect health care in terms of both improving patient care and economics. Researchers ought to consider how to design molecular imaging probes that fully exploit technologic innovations 
as they are being developed-not $10 \mathrm{y}$ after they are commercialized. The field also has to identify and put into action new discovery technologies and evaluative programs that can simplify the process of finding, validating, and properly testing new molecular imaging probes. It simply takes too long to move from discovery to use. The key to the field's future is to shorten that time.

Nuclear medicine, molecular imaging, and probe research efforts are expanding at such a rate that the number of studies published in a single year represents an enormous volume of information. Rather than even attempt a comprehensive review, this article aims to highlight unique approaches, promising developments, and the opportunities that can be unearthed by forming new bridges between disciplines. The focus is on 3 core areas as representative examples. The first area is the development of probes for emerging imaging technologies; the second is strategies to exploit these technologies and probes for improving breast cancer detection, biopsy, surgery, and pathology; and the third is some key challenges of probe development and the strategies that scientists are taking to address these challenges. It is critically important for the scientific community at large to understand these issues so that new approaches from outside the immediate field can be used to enhance probe development.

\section{ORGAN- AND DISEASE-SPECIFIC CAMERAS}

Over the past several years, there has been increasing activity in the literature around the development of dedicated imaging systems (1-3). This activity has translated into the commercialization of new imaging devices for cardiology and oncology applications. The field is coming full circle in that some of the original scanners, particularly for PET, were developed for specific applications, with the one notable example being brain imaging (4-6). One technology that has emerged recently and is garnering significant interest from clinicians and probe developers is dedicated breast imaging systems.

Mammography is an unquestionably important frontline screen for breast cancer and has had a positive impact on overall survival. Mammography, however, fails to detect about $10 \%-15 \%$ of all breast cancers (7-9). It is suboptimal for women with radiodense and fibroglandular breasts, and its sensitivity drops significantly from about $71 \%-96 \%$ to $48 \%-$ $63 \%$ in those cases. This reduced sensitivity in such cases is made even more problematic in that women with dense breasts tend to be at higher risk for breast cancer. There is also growing concern about the high false-positive rate of mammography, which is causing significant numbers of unnecessary biopsies to be performed. In addition to the needless anxiety that a false-positive finding causes patients, it also increases health care costs and the risk of infection. Better imaging strategies that can address this issue are critically needed.

Scintimammography would seem to be an obvious solution to some of the above-mentioned issues. In scintimammography, the image shows uptake of a tracer into breast canceruptake that should be independent of breast density (or at the least much less dependent on breast density than are mammographic findings). Unfortunately, conventional $\gamma$-cameras have a limited ability to reliably detect tumors that are smaller than $1 \mathrm{~cm}(10)$. This limitation has, however, provided the impetus for physicists and engineers to develop dedicated breast imaging systems.

Several $\gamma$-camera-based systems have been developed specifically for breast imaging. One that has received attention was based on work done at the Mayo Clinic; the camera system is based on cadmium zinc telluride detectors (Fig. 1) $(3,11)$. The intrinsic resolution for cadmium zinc telluride-based breast imaging systems is on the order of 5-7.5 mm (9). Figure 2 shows a mammogram of a patient with a $20-\mathrm{mm}$ invasive ductal carcinoma that is also visualized on molecular breast imaging using sestamibi as the tracer. What is also evident, and particularly impressive, is an additional $10-\mathrm{mm}$ cancer that is seen only on molecular breast imaging.

Another important driving force for the development and translation of new breast cancer imaging probes is the desire to use molecular breast imaging to derive molecular information from the images to guide biopsy, treatment selection, and treatment monitoring. Because targeted therapies using biotechnology products are extremely expensive, it is imperative that the appropriate molecular imaging methods be available to stratify patients toward the best treatment option.

There has been a tremendous amount of work done on the development of SPECT agents that target specific molecular signatures found on breast cancer. Two areas that are particularly active involve the development of ${ }^{111} \mathrm{In}$ - and ${ }^{99 \mathrm{~m}}$ Tc-labeled antibodies and peptides.

Human epidermal growth factor receptor $2 \mathrm{HER} 2 / \mathrm{neu}$ (ErbB-2) is a target for developing molecular imaging probes for breast cancer because of its use in prognosis and because it is a target for therapy using trastuzumab (12). Specific imaging probes based on radiolabeled antibodies and associated fragments have shown success in early studies $(13,14)$. Several groups have developed radiolabeled trastuzumab, but one of the challenges in this approach, which will apply in general to probes derived from therapeutic antibodies, is that the therapy agent can compete with the radiolabeled form for the target of interest.

An innovative strategy that is being investigated is to target complementary proteins. McLarty et al., for instance, showed that micro-SPECT/CT (Fig. 3) of HER2overexpressing breast cancer could be achieved using ${ }^{111}$ In-labeled pertuzumab, a second-generation antibody intended to block HER2 dimerization, which binds to domain II of the receptor. Because this domain is different from that to which trastuzumab binds (i.e., domain IV), this type of molecular imaging of HER2 expression should be possible in patients who are receiving trastuzumab therapy. 


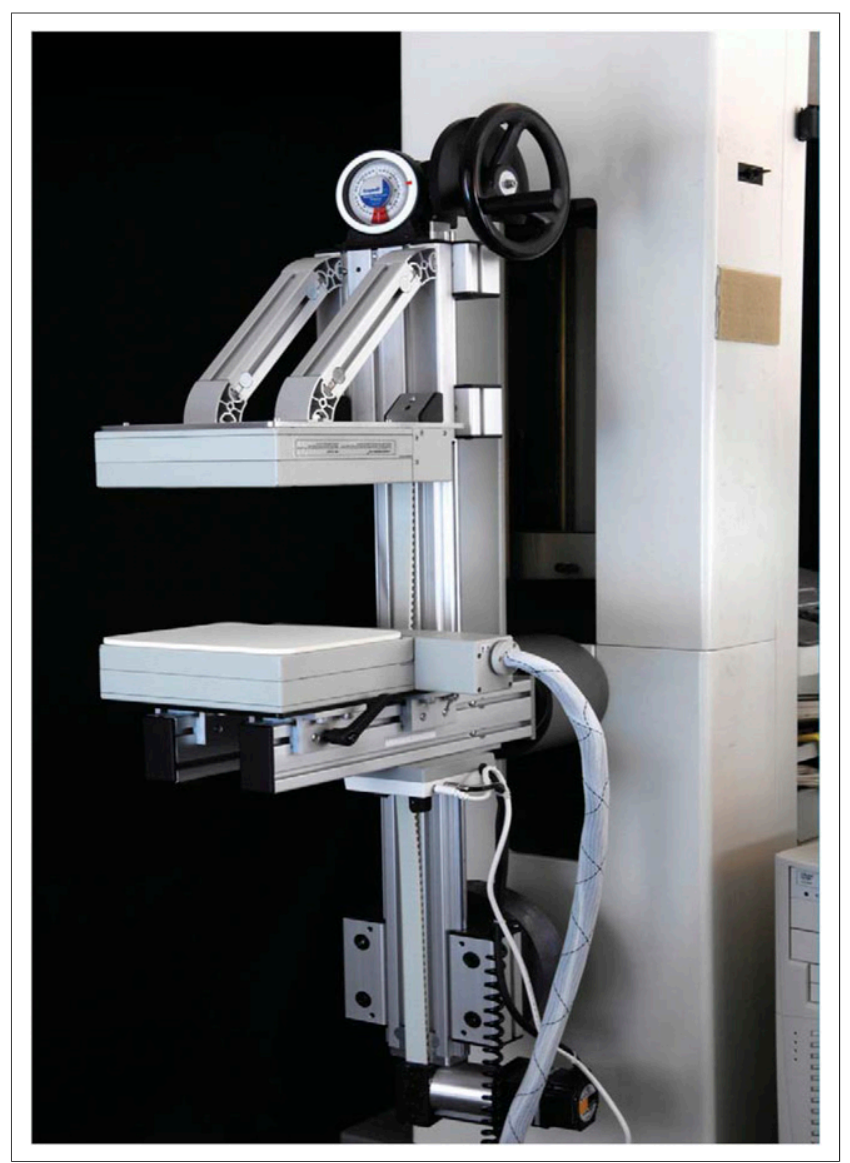

FIGURE 1. Cadmium zinc telluride-based molecular breast imaging camera. (Reprinted with permission of (79).)

With the increasing understanding of the proteome, the development of other complementary targeting probes should be expected to increase over the next $5 \mathrm{y}$.

Peptide-based agents are perhaps one of the most active areas for targeting SPECT (and therapeutic) radionuclides to specific receptors. One particularly attractive example is the work reported by Bach-Gansmo et al., who demonstrated how a novel probe coupled with new imaging technologies can yield exciting results (15). In this work, a technetium-labeled peptide that has high affinity for the $\alpha_{v} \beta_{3}$-integrin was used in a breast cancer imaging study that applied both conventional scintimammography and a dedicated breast imaging system (16). The study showed that invasive ductal carcinomas ranging from 6 to $20 \mathrm{~mm}$ were well visualized. Images derived from conventional scintimammography were able to locate tumors in certain cases; however, even with an early-generation molecular breast imaging camera it was possible to find tumors as small as $7 \mathrm{~mm}$ (invasive ductal carcinoma) that were barely visible using conventional imaging methods (Fig. 4).

Dedicated breast imaging is not solely the purview of $\gamma$-camera-based technologies, as there has been increasing work on developing PET scanners specifically for breast imaging. Whole-body ${ }^{18}$ F-FDG PET has limited sensitivity for breast cancer and is not well suited for detecting small,

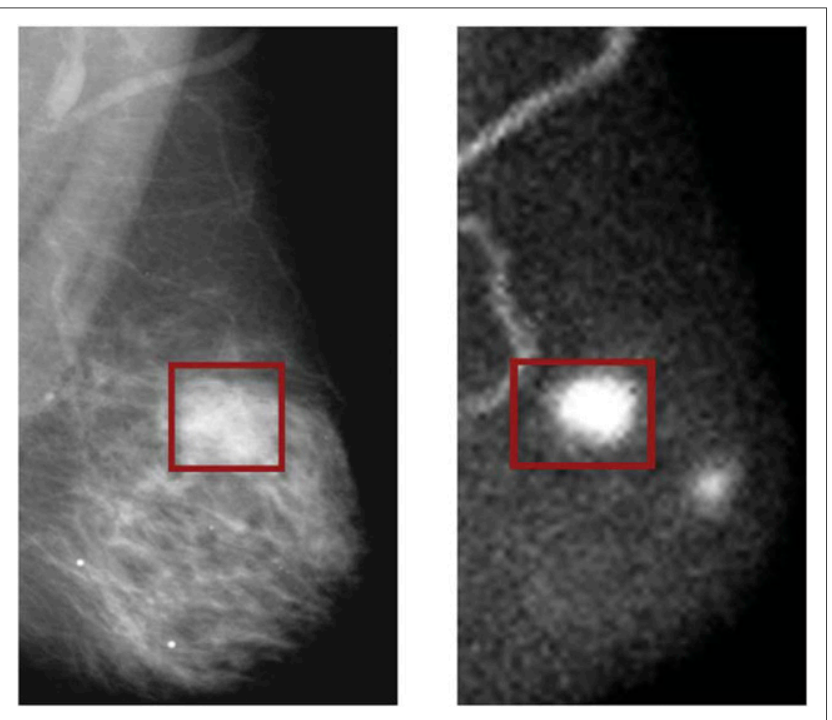

FIGURE 2. Mammogram (left) and molecular breast imaging scan (right) of same patient. Twenty-millimeter cancer (red box) can be seen on both images, but only molecular breast image (using sestamibi) shows additional 10-mm lesion. (Reprinted with permission of (80).)

well-differentiated in situ breast carcinoma (17). Dedicated PET systems can address some of these limitations and offer higher spatial resolution, shorter imaging times, reduced attenuation, and a compact footprint, and they can be integrated with biopsy procedures.

One commercially available system is the PEM (positron emission mammography) scanner (Naviscan). Figure 5 shows a 40-y-old patient who after mammography and ultrasound and MRI was diagnosed with invasive ductal carcinoma in the left inferior breast. Positron emission mammography using ${ }^{18} \mathrm{~F}$-FDG actually showed that there was bilateral cancer. One of the advantages that PET has over $\gamma$-camera systems is that there exists a library of PET probes that can be used in conjunction with dedicated breast PET scanners (18-21). Agents have been developed for targeting proliferation, angiogenesis, hypoxia, and the estrogen receptor. $16 \alpha{ }^{-18} \mathrm{~F}$-fluoro- $17 \beta$-estradiol is particularly interesting given the importance of hormone status in determining the expected response to therapy $(22,23)$. Clinical studies with this agent are ongoing and involve comparing uptake with that of ${ }^{18} \mathrm{~F}-\mathrm{FDG}$ in terms of response of therapy $(24,25)$.

The development of the next generation of PET probes for breast cancer is an active area of research. Targeting strategies include radiolabeled antibodies, antibody fragments, small molecules, and peptides using a wide array of different PET isotopes and creative labeling and bioconjugate chemistry methods. Although each of these areas has been reviewed lately (26-31) or deserves its own dedicated reviews, one unique and emerging area involves exploiting genetic engineering.

Genetic engineering is a rapidly maturing field that can be applied to identify and manufacture high-affinity 
FIGURE 3. McLarty and Reilly showed that micro-SPECT/CT of HER2-overexpressing breast cancer could be achieved using ${ }^{111}$ In-labeled pertuzumab. Because this domain is different from that to which Herceptin binds, it should be possible to perform this type of molecular imaging of HER2 expression even in patients being treated with trastuzumab. (Courtesy of Raymond Reilly, University of Toronto.)

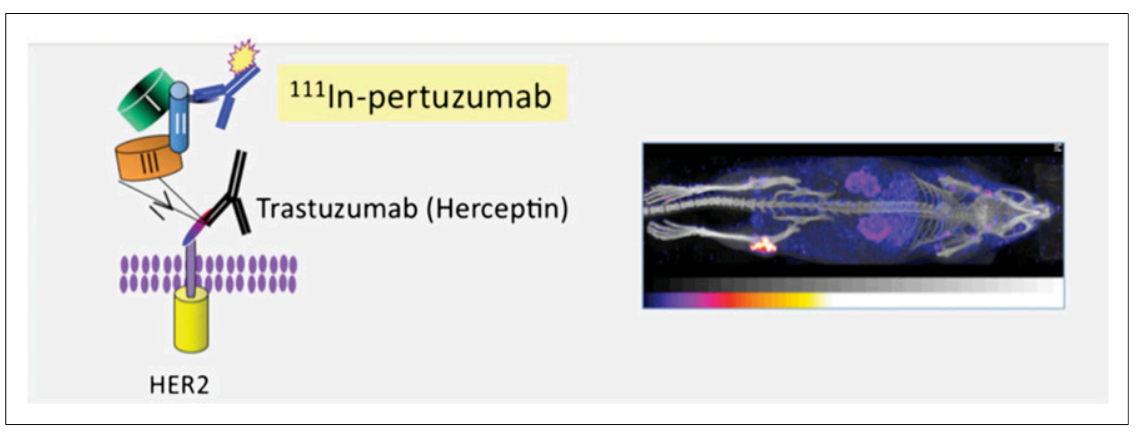

targeting vectors. Affibody molecules (Affibody AB), for example, are attractive targeting agents because they can be considered to be somewhere between an antibody and a peptide, having the high affinity of one and the good pharmacokinetics of the other (32). Affibody molecules can be produced using recombinant bacteria or by conventional peptide synthesis, providing a reasonable pathway for manufacturing sufficient quantities for both research and clinical trials.

A radiolabeled Affibody for $H E R 2$ was recently reported by Kramer-Marek et al. (33). Images of the ${ }^{18} \mathrm{~F}$-labeled form of the Affibody (Fig. 6) in mice bearing SKOV-3 tumors showed high uptake in the tumor as early as $20 \mathrm{~min}$ after injection. Early liver and kidney uptake, which has generally plagued peptide- and antibody-based imaging and therapy agents, was high only during the first hour and dropped noticeably over time. The agent cleared via the renal pathway with a half-life of approximately $36 \mathrm{~min}$, which is far shorter than the half-lives of radiolabeled antibodies and antibody fragments.

The widespread availability of ${ }^{18} \mathrm{~F}$ makes approaches such as these attractive compared with antibody-based strategies, which require the use of longer-lived and often less readily available PET isotopes. In addition, because of advances in genetic engineering, it is possible to produce libraries of Affibody-type vectors that can be screened against different targets that are upregulated in breast or other cancers. The major issues with this vector surround the cost of finding

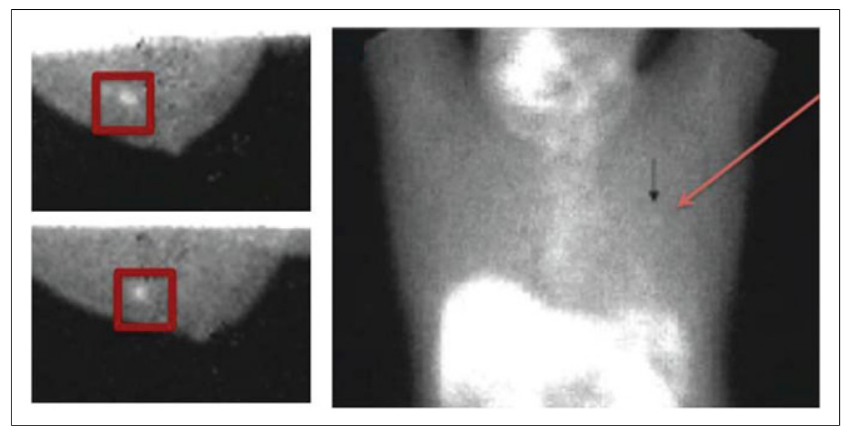

FIGURE 4. Images of peptide-based agent ( ${ }^{99 m}$ Tc-NC100692) using both dedicated breast imaging system and conventional scintimammography. Red boxes delineate 7-mm invasive ductal carcinoma that is barely visible on scintimammography (arrow). (Reprinted with permission of (16).) leads and intellectual property barriers that could limit its full exploitation. Affibody-based strategies will also benefit from the development of new radiolabeling and purification technologies designed specifically for proteins of this size.

\section{HYBRID IMAGING SYSTEMS}

New hybrid imaging technologies, much like diseaseor organ-specific cameras, are driving advances in probe development. The combination of PET and SPECT cameras with CT is now an industry standard; consequently, the innovation focus has shifted toward the development of hybrid PET/MRI, and to a lesser extent SPECT/MRI, systems as preclinical and clinical imaging tools. Researchers are now taking advantage of these emerging technologies to develop probes that can make use of the combined modalities. Multimodality probes are agents that are detectible by more than one imaging method (34-36). Most agents have, to date, been derived from nanoparticles and include combined optical and MRI contrast agents; however, there is an increasing number of reports on nuclear-MRI and nuclear-optical agents.

Examples include coated iron-oxide nanoparticles derivatized with a targeting vector and a chelate for radiolabeling. For example, iron-oxide nanoparticles derivatized with arginineglycine-aspartic acid peptides for targeting angiogenesis and

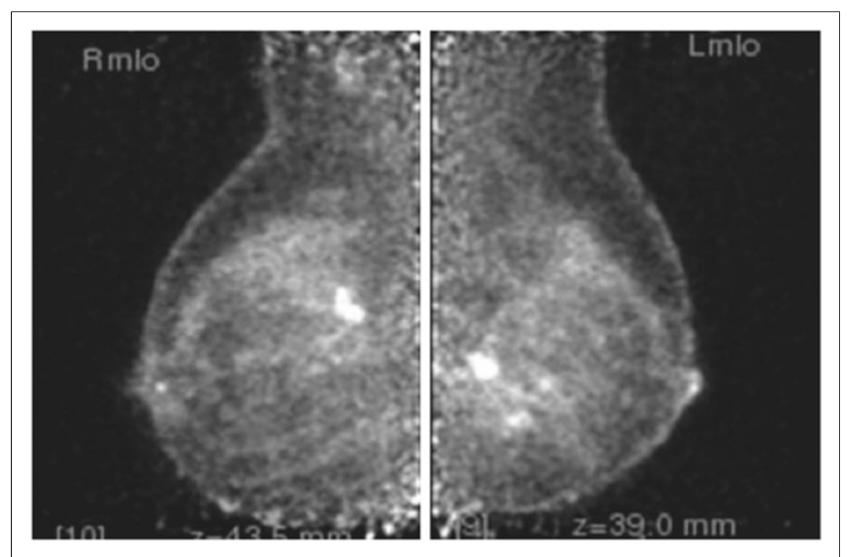

FIGURE 5. ${ }^{18} \mathrm{~F}-\mathrm{FDG}$ positron emission mammography scan of 40-y-old woman whose first mammogram showed area of increased density in left inferior breast. Positron emission mammography scan shows invasive ductal carcinoma in both breasts. (Courtesy of Jacquelyn Jordan Gray, Naviscan, Inc.) 


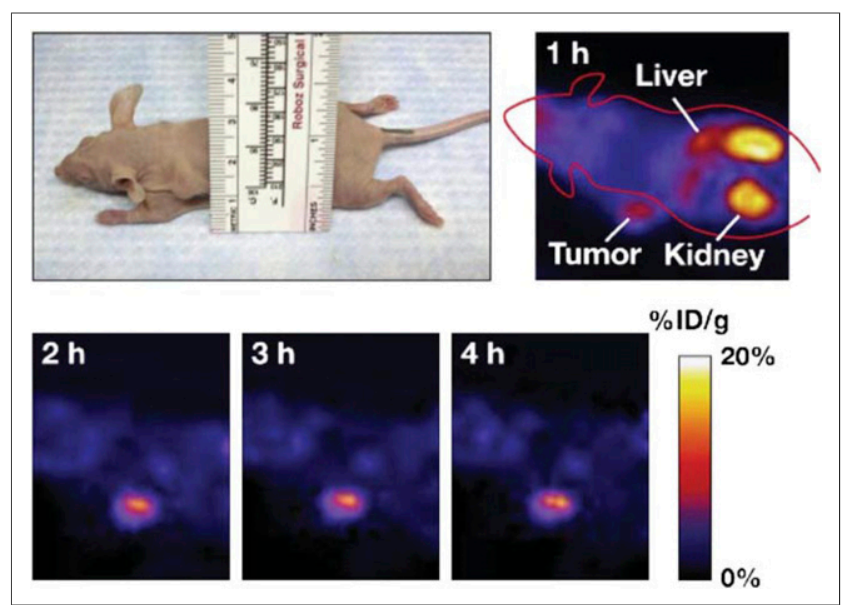

FIGURE 6. Small-animal PET images of ${ }^{18} \mathrm{~F}$-labeled Affibody for HER2 in mice bearing SKOV-3 tumors. (Reprinted with permission of (33).)

DOTA chelates for binding ${ }^{64} \mathrm{Cu}$ were prepared by Lee et al. (37). The nanoparticles were shown to bind $\alpha_{\mathrm{v}} \beta_{3}$-integrinexpressing cells in vitro and were shown to have specific uptake in nude mice bearing human U87MG tumors. This uptake could be visualized on preclinical PET and MRI scanners, and blocking studies confirmed that uptake was due to active targeting (Fig. 7).

Sun et al. showed that block copolymers can be used to prepare nanomaterials for imaging (38). In this work, the metal chelator DOTA was grafted onto block copolymer precursors from which shell cross-linked knedellike nanoparticles were assembled. The composition of hydrophilic and hydrophobic blocks can be varied, making it possible to control the overall properties of the material and the number of sites bearing a chelate and targeting vector. As the optimal features for nanoparticle-based agents continue to be refined, the synthetic versatility and potentially less toxic nature of polymer-based systems make this approach appealing.

There is general concern over the toxicity of nanoparticles and the associated imaging agents. The cost of evaluating the safety of new agents could be significant, creating a barrier to translation that is higher than for traditional radiopharmaceuticals. There is also need for robust synthetic methods for the preparation of nanoparticle-based imaging probes, which must be manufactured to the standards expected of traditional molecular imaging probes, and the development of strategies to prevent sequestering by the reticuloendothelial system. The latter is a key issue for several SPECT/MRI nanoparticle probes including indium-labeled iron oxide agents (37). These are not unsolvable problems; they are simply challenges that require creative solutions to address.

Probe developers are now developing multimodality agents for hybrid technologies beyond CT and MRI. Probes that can be used for both nuclear-optical and nuclear- ultrasound imaging have been reported recently. Optical imaging is attractive because of its high sensitivity, and it offers a way to validate new targeting strategies and link diagnostic imaging with pathology. Pathology laboratories are increasingly using fluorescent markers and confocal microscopy to evaluate tissue samples, providing a new area of application for molecular imaging probes.

Optical and nuclear hybrid imaging probes can be developed using radiolabeled luminescent particles or quantum dots $(39,40)$. Another strategy involves derivatizing a targeting vector with a radiolabeled prosthetic group and a fluorescent tag. Bhushan et al. developed a dual-modality SPECT/near-infrared fluorescent probe for the detection of breast cancer microcalcifications (41). A bisphosphonate ligand was derivatized with a ${ }^{99 \mathrm{~m}} \mathrm{Tc}-\mathrm{N} 3 \mathrm{~S}$ chelate complex and IRDye $800 \mathrm{CW}$. The intraoperative near-infrared fluorescence image and SPECT/CT images show high uptake in the skeleton and microcalcified tumors.

Preclinical ultrasound systems have been developed to support animal imaging studies in which the instruments generate images of exquisite resolution (Fig. 8). With hybrid ultrasound-nuclear probes it would be possible to combine real-time high-resolution ultrasound images with the quantitative nature of nuclear methods. Another attractive feature of ultrasound involves image tissue sonication (42). This technique can be used to temporarily increase the permeability of membranes, which could be used to increase the regional uptake of SPECT and PET probes, leading to enhanced target-to-nontarget ratios.

The first report of a dual-modality PET-ultrasound probe was in 2008 (43). A perfluorocarbon gas-filled microbubble surrounded by a lipid shell coated with streptavidin provides a convenient platform for attaching biotinylated antibodies. To make a hybrid derivative, the ${ }^{18} \mathrm{~F}$ tag was first conjugated to a rat antimouse vascular endothelial growth factor receptor 2 antibody before the vector was linked to the ultrasound bubble. The distribution of the agent showed high liver and spleen uptake and was different from that of the labeled antibody alone.

Our group took a slightly different approach in which a technetium chelate conjugated to biotin was labeled with the $\left[\mathrm{Tc}(\mathrm{CO})_{3}\right]^{+}$core and conjugated to a streptavidincoated microbubble. This platform, which includes in situ purification to remove any residual unlabeled and labeled biotin-containing ligands, was designed so that any biotinylated antibody can be linked to the SPECTultrasound visible bubble (44).

Despite the excitement around multimodality imaging probes, several concerns that have been raised need to be addressed before these agents can fulfill their promise. First, performance is often sacrificed when technologies are combined (45). In the case of PET/MRI, as an example, if a large quantity of agent is needed to get sufficient MRI contrast, there will likely be a deleterious impact on the target-to-nontarget ratios in the PET images. 
FIGURE 7. Small-animal MRI and PET scans of subcutaneous $a_{v} \beta_{3}$-expressing glioma xenografts administered coated iron oxide multimodal probe derivatized with cyclic arginine-glycine-aspartic (RGD) acid peptides and DOTA chelating groups. Images involving targeted agent, control particles, and specific blocking experiments in nude mice are shown.

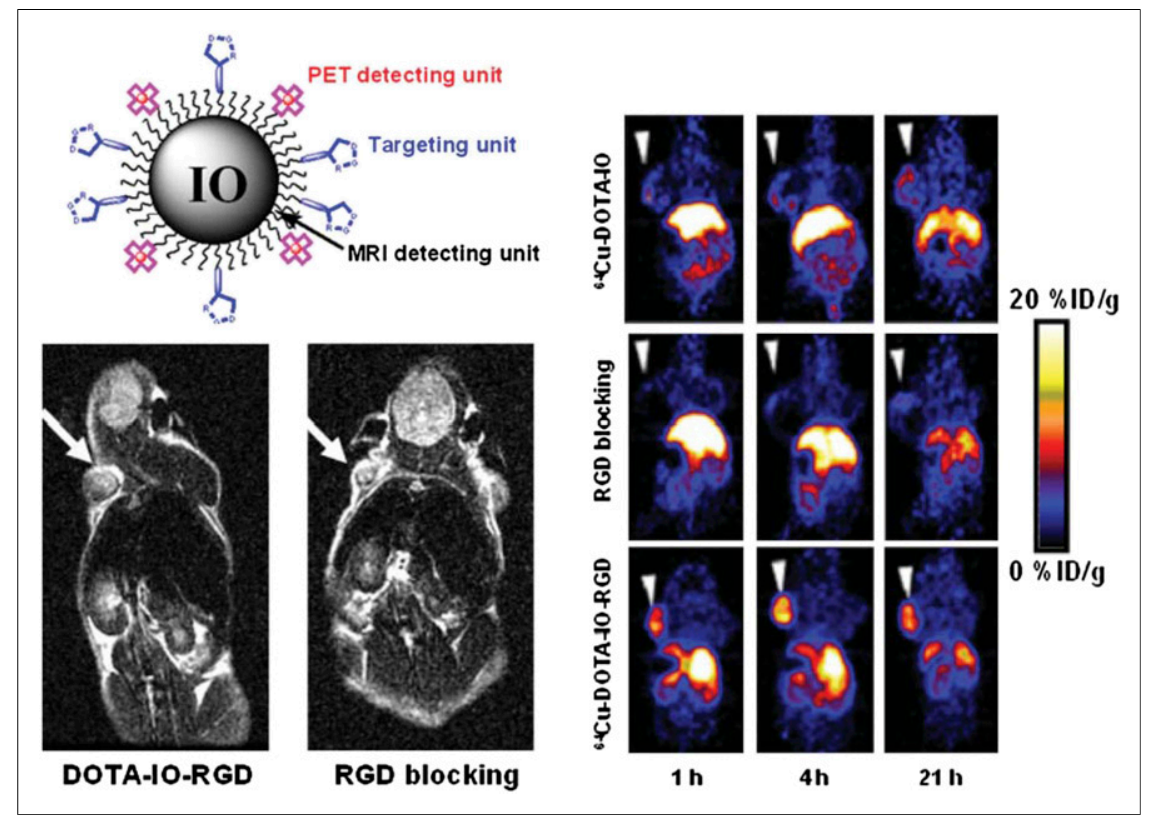

\section{LINKING DISCIPLINES THROUGH MOLECULAR IMAGING PROBES: ENHANCING BREAST CANCER TREATMENT}

Three important components of effective treatment of breast cancer are biopsy guidance, surgical margin determination, and pathology. Dedicated and hybrid imaging scanners in conjunction with the appropriate molecular imaging probes offer the opportunity to address some of the key issues within these areas (46).

With respect to biopsy, ultrasound and MRI are now used almost routinely for anatomic guidance. The opportunity to use molecular imaging probes for biopsy is appealing because it affords the use of molecular signatures to guide sampling (47). Adequate spatial resolution to do this can now be achieved using the aforementioned dedicated breast

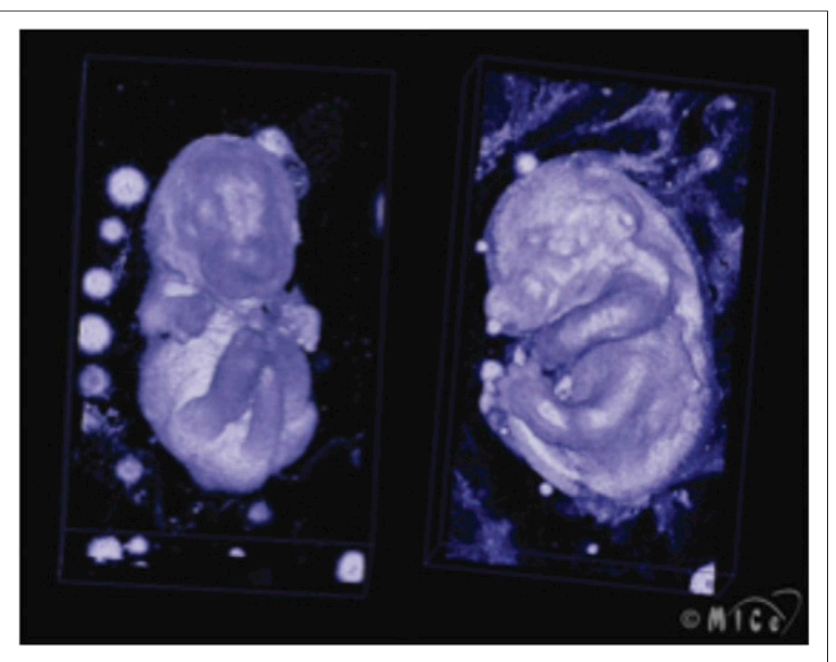

FIGURE 8. Ultrasound image of mouse embryo. (Courtesy of Stuart Foster, Sunnybrook Health Sciences Centre.) imaging systems. For example, positron emission mammography and ${ }^{18} \mathrm{~F}-\mathrm{FDG}$ uptake in tumors have been used to guide biopsy and sampling; the cores can be evaluated for regions of high, medium, and low levels of ${ }^{18}$ F-FDG uptake (Fig. 9). This is an exciting area for future development, particularly when combined with probes that target proteins whose expression guides the choice of therapy. Key examples would include estrogen receptor- and HER2-targeting agents, for which expression of the targets is already used to direct decision making toward the optimal intervention.

Moving from biopsy to surgery, there is a need for better technologies for margin determination, which is becoming increasingly important because of the growing number of breast-conserving surgical procedures that are being used. Failure to obtain clear margins is one of the leading risk factors associated with reoccurrence and reoperation, for positive margins can occur in up to $50 \%$ of the cases (48).

To help guide surgical procedures, new intraoperative $\gamma$-probes have been developed. These are designed to help surgeons detect tumor cells at or near the margin of resection. Several new detector probes have been developed to detect technetium-based agents, but there have also been reports on the use of probes for iodine-based agents, including metaiodobenzylguanidine $(49,50)$.

Hand-held PET probes are also available that can detect positrons emitted by PET tracers (51). Positron probes are particularly interesting because the probe is responsive over a short distance $(1-2 \mathrm{~mm})$. As a result, there is high local sensitivity when moving from tumor to nontumour tissues, eliminating the possibility that uptake in distal organs will confound margin determination. An additional attractive feature is that because collimation is not necessary, these probes can potentially be used in conjunction with minimally invasive surgical strategies. 


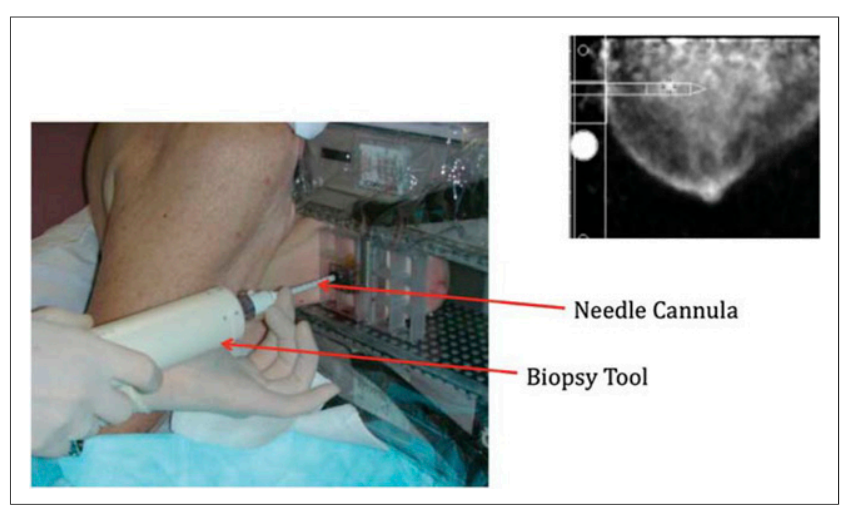

FIGURE 9. Positron emission mammography-guided biopsy based on uptake of ${ }^{18} \mathrm{~F}-\mathrm{FDG}$. (Courtesy of Jacquelyn Jordan Gray, Naviscan, Inc.)

Much like surgery and biopsy, tissue sample analysis can benefit in throughput, accuracy, and reproducibility using molecular imaging probes, with a resulting major impact on cancer care. The development of new molecular imaging probes for pathology is an emerging area in which complementary radioactive and fluorescent probes can be used to enhance early detection and the evaluation of tumor samples, respectively.

One approach to preparing this type of probe is to develop the chemistry that enables one to derivatize a cancer-seeking vector separately with a radiolabeled prosthetic group and a fluorophore suitable for imaging tissue samples. A convenient way to achieve such an outcome is to use click chemistry $(52,53)$. At the heart of click chemistry is the Huisgen cycloaddition reaction between alkyne and azide. The reason the click reaction has become so popular for probe developers is that these 2 functional groups do not tend to react with other functional groups found on biovectors; it therefore is possible to carry out selective coupling reactions. Since the first use of click chemistry to tag peptides with ${ }^{18} \mathrm{~F}(54)$, there has been a tremendous amount of work in this area leading to an array of novel labeling strategies (55-58).

In one example, click chemistry was used to elaborate a single targeting vector into an array of molecular imaging probes for use with different imaging modalities (59-62). Schibli et al. (59) developed a library of probes for the folate receptor because of its increased expression in several different cancers. Products included an ${ }^{18} \mathrm{~F}$-labeled derivative for PET, a technetium chelate conjugate derived from the triazole core for SPECT, a fluorine-rich group for multinuclear MRI, a dye for optical or nearinfrared imaging, and 2 other chelates for developing different types of SPECT agents. The appeal is that one targeting vector was used to create multiple agents, including complementary optical and nuclear probes. This general approach to probe development will likely be used more extensively given that the nature of the chelate and spacer can have a profound impact on the distribution and clearance of a probe.
One issue with swapping a radionuclide-containing prosthetic group with a fluorophore on a given targeting vector is that the products have different structures, charges, and lipophilicities. Although the affinities of the 2 probes for the target of interest may be similar, their biodistribution profiles will likely be different. For correlating in vivo imaging with pathology, the ideal case would be for the structures of the fluorescent and radiolabeled prosthetic groups to be identical.

Our group reported the development of a chelate system whereby the nonradioactive rhenium complex was fluorescent (63). The ${ }^{99 \mathrm{~m}} \mathrm{Tc}$ analog, because of the lanthanide contraction, has the same structure as the rhenium complex and can be used for in vivo imaging. The single-amino-acid chelate-quinoline (SAACQ) ligand is a convenient platform for developing molecular imaging probes because it can be incorporated into peptides as if it were a natural amino acid. The rhenium complex is inert, and it too can be incorporated into peptides, making it possible to prepare fluorescent agents using standard automated peptide synthetic methods. If a viable fluorescent agent is found, the analog containing the free uncomplexed ligand can be prepared and then labeled with ${ }^{99 \mathrm{~m}} \mathrm{Tc}$ and in vivo imaging studies performed.

The SAACQ system has been used to prepare complementary probes for imaging the formyl peptide receptor (63), imaging amyloid plaques (64), and tagging stem cells (neurospheres) (65). More recently, we have shown that the rhenium complex of insulin conjugate can be administered to animal models, the tissues excised, and the fluorophore imaged using confocal microscopy even when using low concentrations of the probe (65). These results indicate that it should be possible to use a ${ }^{99} \mathrm{~m}$ Tc-SAACQderived agent in conjunction with a dedicated imaging breast system for early detection and biopsy of breast cancer and to use the complementary fluorescent rhenium agent to support pathologic analysis of excised tissue. In parallel, the probes could be used to support surgical guidance via intraoperative $\gamma$ - or fluorescent probes.

\section{CHALLENGES IN PROBE DEVELOPMENT}

The foremost challenge in molecular imaging probe development is to identify strategies to increase target binding while reducing nonspecific binding. Low-molecular-weight targeting vectors are often either cleared too rapidly or prematurely metabolized, leading to unwanted uptake in the liver, kidney, or gut. Probes derived from large biovectors tend to have significant hepatobiliary or kidney retention as well. Residual uptake in nontarget organs detracts from the overall utility of molecular radioimaging.

Researchers are beginning to develop innovative solutions to these problems. The most common approach is to attach hydrophilic prosthetic groups or polyethylene glycol spacer chains (66). More recent work has involved the use of hydrophilic dendrimers, which are highly branched and structurally perfect macromolecules. Dendrimers can be 
functionalized at the periphery or at the core, providing flexible platforms for modifying pharmacokinetics and reducing nonspecific binding.

Parrott et al. recently reported a polyester dendrimer that contained a $\mathrm{Tc}(\mathrm{I})$ chelate at the core (67). This ligand could be labeled with ${ }^{99 \mathrm{~m}} \mathrm{Tc}$ using a microwave labeling method that produced the agent in less than $15 \mathrm{~min}$ in high yield. Biodistribution data and dynamic small-animal imaging studies demonstrated that the dendrimers (generations 57), unlike the chelate complex alone, which has high uptake and retention in the gut, clear extremely rapidly and end up in the bladder in less than $15 \mathrm{~min}$.

Almutairi et al. showed how dendrimers can also be used for PET of angiogenesis (68). A biodegradable dendrimer core was linked to a targeting vector derived from cyclic arginine-glycine-aspartic acid and radiolabeled with ${ }^{76} \mathrm{Br}$ $\left(\beta^{+}\right.$, half-life $\left.=16.2 \mathrm{~h}\right)$. By incorporating multiple targeting vectors there was a nearly 50 -fold increase in binding affinity for the target over one targeting vector alone. Using a murine hind limb ischemia model, the agent showed selective and high uptake at sites of angiogenesis. New strategies such as the use of dendrimers for modifying both pharmacokinetics and affinity for the target, that are also sufficiently flexible to use with different targeting vectors, are needed to overcome the pressing issue of high nontarget tissue uptake.

A second challenge, which is unique to radionuclidebased molecular imaging probes, involves production. Most production methods for radiopharmaceuticals involve highperformance liquid chromatography purification or instant kits. The latter is particularly problematic for molecularly targeted agents in that instant kits were not designed for the molecular imaging era. For technetium kits, for example, the ligand to be labeled is generally present in a large excess, compared with the radiolabeled product; the unlabeled ligand, which generally retains high affinity for the target, will compete with the labeled species. This is not to say that the maximum effective specific activity will always yield the highest target-to-nontarget ratios, as some carrier can prevent binding to circulating proteins or proteins in nontarget tissues. What is needed is a way to select the optimal effective specific activity.

Several solutions have been developed to address this issue. The simplest approach involves developing more reactive chelates that do not have to be used in a large excess to achieve a high radiochemical yield. Microfluidic technologies are also being used to reduce the ratio between isotope and the actual agent and to incorporate in-line purification methods into the production process. Two other technologies, which have been developed for radiohalogens, are solid-phase and fluorous-phase labeling $(69,70)$. The general procedure involves preparing aryl stannane derived from the agent to be labeled, in which the tin atom is linked to a cross-linked polymer or a fluorine-rich group. On reaction with iodine and oxidant, the tin-aryl bond is cleaved. The residual unreacted starting material can be separated from the desired radiopharmaceutical by filtration (solid-phase system) or by passing the mixture through a fluorous-solid-phase extraction cartridge (fluorous-phase labeling). In the latter, the high affinity of fluorine-rich molecules for each other results in a chemoselective filtration that produces the desired products in high effective specific activity (Fig. 10). These approaches have been used to produce several agents, including fialuridine, iodeoxyuridine (71), and metaiodobenzylguanidine (72), and have recently been applied to the production of ${ }^{18} \mathrm{~F}$-labeled compounds (73).

Another acute issue facing the probe development field involves isotope shortages. With the protracted scarcity of ${ }^{99 \mathrm{~m}} \mathrm{Tc}$, the probe-development and nuclear medicine communities have been developing and evaluating surrogate agents. One of the most active areas has been cardiac imaging, in which there has been increased interest in using ${ }^{201} \mathrm{Tl}$ and ${ }^{82} \mathrm{Rb}$ and active development of new ${ }^{18} \mathrm{~F}$, ${ }^{68} \mathrm{Ga}$, and ${ }^{64} \mathrm{Cu}$ agents (74-77). In nearly all countries, the number of $\gamma$-cameras far exceeds the number of PET cameras. We can therefore expect to see an increase in activity around the development of SPECT agents using ${ }^{123} \mathrm{I}$ and ${ }^{111}$ In either to replace existing technetium agents or to act as surrogates during times of emergency.

${ }^{68} \mathrm{Ga}$ deserves special mention in this context because of its availability via a ${ }^{68} \mathrm{Ge}$ generator $(77,78)$. The generator system eliminates the need to have a cyclotron on-site or near the imaging facility. Like ${ }^{99 \mathrm{~m}} \mathrm{Tc},{ }^{68} \mathrm{Ga}$ requires the use of a chelator that allows one to study the work done on creating targeted technetium agents and apply the lessons learned to the development of gallium-based agents. Much progress has been made in this regard, and agents such as ${ }^{68} \mathrm{Ga}$-DOTATOC are yielding striking PET images clearly demonstrating the future potential of ${ }^{68} \mathrm{Ga}(78)$.

The fourth challenge is moving discoveries from the laboratory to the clinic and out to the market. The Canadian experience has been that there was commercial and clinical
FIGURE 10. Fluorous labeling method. After treatment of fluorine-rich starting material with radioiodine and oxidant, unreacted starting material and fluorous byproducts can be separated from product using solid-phase extraction cartridge containing fluorine-rich bonded phase (fluorous solid-phase extraction, or FSPE). (Reprinted with permission of (70).)

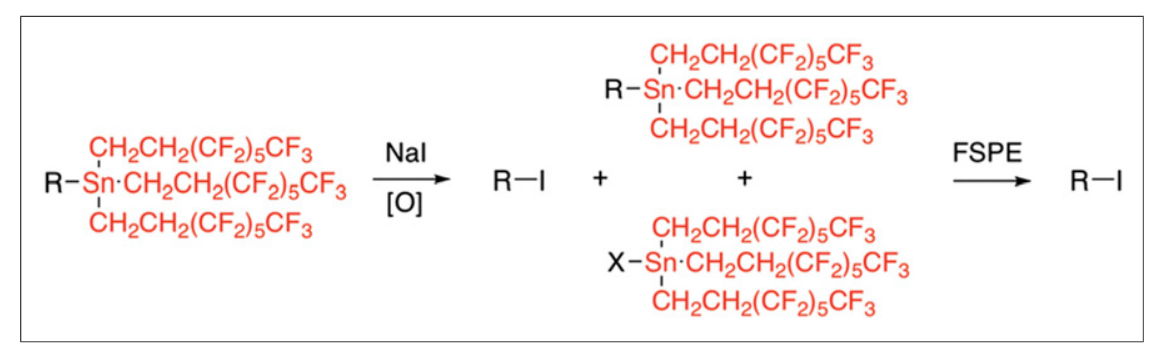


demand for new agents but few ways to complete the preclinical safety and regulatory work needed to bridge the research laboratory and the clinic. This problem was compounded by the lack of funding for clinical trials of sufficient size to properly evaluate novel molecular imaging probes. To address this issue, the Centre for Probe Development and Commercialization (www.imagingprobes.ca) was created with funding from federal and provincial governments and industry partners. The objectives of the center are to validate, translate, and commercialize innovative imaging probes and to make probes available for clinical studies and basic research and development. Similar initiatives and expanded multicenter trial capabilities are being considered and implemented around the world and are crucially important to securing the future of the field and to ensuring that the basic science surrounding molecular imaging has a positive impact on patient care.

\section{CONCLUSION AND LOOKING TO THE FUTURE: A 2020 (20/20) VISION OF PROBE DEVELOPMENT}

So long as the field looks to expand its horizons by collaborating with other disciplines, the future for nuclear medicine and molecular imaging is bright. In the future, it is likely that probes will be specifically designed to have multiple uses including early detection, surgical biopsy guidance, and automated pathologic analysis of cancer biopsy and surgical samples. There will be an increase in the use of organ- and disease-specific scanners, which will soon become hybrid imaging systems that include CT capabilities. Isotope supply redundancy will be achieved through expansion of reactor and cyclotron production programs and through collaborative probe development and clinical trial efforts. This goal can best be achieved through coordinated international partnerships-an important opportunity that is currently in its infancy.

Senior researchers should encourage young scientists to explore unique directions and not listen to statements such as "we have enough ligands or agents." It is always easier to state that today's technology is sufficient than it is to come up with tomorrow's innovation. To avoid stagnation, the field must stop recycling the same reliable targeting vectors, particularly those that have not made it to the clinic despite years of study. Groups involved in genetic and proteomic research are producing vast sums of data around potential new targets that are associated with disease onset and progression. The nuclear medicine and probe development fields must learn to exploit this information rapidly.

Basic chemistry and access to well-trained chemists will continue to be a critically important part of nuclear medicine. New isotope production and separation methods, novel chelate systems, creative labeling, and bioconjugate chemistry strategies are needed to generate the agents of tomorrow. Many of these innovations will require forming bridges outside the fields of radiochemistry and nuclear medicine to emerging areas such as chemical biology.
Those willing to cross the bridge to these new collaborations will yield the major breakthroughs of the future.

\section{ACKNOWLEDGMENTS}

I acknowledge the helpful assistance of Kathryn Blais, Dr. Laura Harrington, and Alicia Jeanveau in preparing the manuscript. The Valliant Research Group receives funding from the Ontario Institute of Cancer Research (OICR), Cancer Care Ontario (CCO), the Ontario Research Fund (ORF), the Canadian Institutes of Health Research (CIHR), the Natural Sciences and Engineering Research Council (NSERC), Molecular Insight Pharmaceuticals (MIP), Biotage Inc., and Pfizer Inc. The Centre for Probe Development and Commercialization receives public funding from the federal government's Centers of Excellence for Commercialization and Research (CECR) program, Cancer Care Ontario, and the Ontario Institute for Cancer Research, with funds from the Ontario Ministry of Research and Innovation. More details can be found at www.imagingprobes.ca.

\section{REFERENCES}

1. Zhou M, Johnson N, Blanchard D, Bryn S, Nelson J. Real world applications of breast-specific gamma imaging, initial experience of a community breast center and its potential impact on clinical care. Am J Surg. 2008;195: 631-635.

2. Gambhir SS, Berman DS, Ziffer J, et al. A novel high sensitivity rapid acquisition single photon cardiac imaging camera. J Nucl Med. 2009;50:635-643.

3. Spanu A, Cottu P, Manca A, Chessa F, Sanna D, Madeddu G. Scintimammography with dedicated breast camera in unifocal and multifocal/multicentric primary breast cancer detection: a comparative study with SPECT. Int J Oncol. 2007;31:369-377.

4. Bronwell GL, Sweet WH. Localization of brain tumors with positron emitters. Nucleonics. 1953;11:40-45.

5. Ter-Pogossian MM, Phelps ME, Hoffman EJ, Mullani NA. A positron-emission transaxial tomograph for nuclear imaging (PETT). Radiology. 1975;114:89-98.

6. Garnett ES, Firnau G, Nahmias C. Dopamine visualized in the basal ganglia of living man. Nature. 1983;305:137-138.

7. Tafra L. Positron emission mammography: a new breast imaging device. J Surg Oncol. 2008;97:372-373.

8. Weinmann AL, Hruska CB, O'Connor MK. Design of optimal collimation for dedicated molecular breast imaging systems. Med Phys. 2009;36: $845-856$.

9. Boyd NF, Guo H, Martin LJ, et al. Mammographic density and the risk and detection of breast cancer. N Engl J Med. 2007;356:227-236.

10. Khalkhali I, Villanueva-Meyer J, Edell SL, et al. Diagnostic accuracy of $99 \mathrm{~m}$ Tcsestamibi breast imaging: multicenter trial results. J Nucl Med. 2000;41:19731979.

11. Hruskaa CB, O'Connor MK. Quantification of lesion size, depth and uptake using a dual-head molecular breast imaging system. Med Phys. 2008;35:1365-1376.

12. Harris L, Fritsche H, Mennel R, et al. American Society of Clinical Oncology update of recommendations for the use of tumor markers in breast cancer. J Clin Oncol. 2007;25:5287-5312.

13. Mankoff DA, Link JM, Linden HM, Sundararajan L, Krohn KA. Tumor receptor imaging. J Nucl Med. 2008;49(suppl 2):149S-163S.

14. Lee JH, Rosen EL, Mankoff DA. The role of radiotracer imaging in the diagnosis and management of patients with breast cancer: part 2-response to therapy, other indications and future directions. J Nucl Med. 2009;50:738-748.

15. Bach-Gansmo T, Danielsson R, Saracco A, et al. Integrin receptor imaging of breast cancer: a proof of concept study to evaluate ${ }^{99 \mathrm{~m}} \mathrm{Tc}-\mathrm{NC} 100692 . \mathrm{J} \mathrm{Nucl}$ Med. 2006;47:1434-1439.

16. Bach-Gansmo T, Bogsrud TV, Skretting A. Integrin scintimammography using a dedicated breast imaging solid-state $\gamma$-camera and ${ }^{99 m}$ Tc-labelled NC 100692. Clin Physiol Funct Imaging. 2008;28:235-239. 
17. Lee JH, Rosen EL, Mankoff DA. The role of radiotracer imaging in diagnosis and management of patients with breast cancer: part 1-overview, detection and staging. J Nucl Med. 2009;50:569-581.

18. Smyczek-Gargya B, Fersis N, Dittmann H, et al. PET with $\left[{ }^{18} \mathrm{~F}\right]$-fluorothymidine for imaging of primary breast cancer: a pilot study. Eur J Nucl Med Mol Imaging. 2004;31:720-724.

19. Beer AJ, Haubner R, Sarbia M, et al. Positron emission tomography using $\left[{ }^{18} \mathrm{~F}\right]$ galacto-RGD identifies the level of integrin $\alpha_{\mathrm{v}} \beta_{3}$ expression in man. Clin Cancer Res. 2006;12:3942-3949.

20. Padhani AR, Krohn KA, Lewis JS, Alber M. Imaging oxygenation of human tumors. Eur Radiol. 2007;17:861-872.

21. Katzenellenbogen JA, Welch MJ, Dehdashti F. The development of estrogen and progestin radiopharmaceuticals for imaging breast cancer. Anticancer Res. 1997; 17:1573-1576.

22. Bénard F, Turcotte E. Imaging in breast cancer: single photon computed tomography and positron-emission tomography. Breast Cancer Res. 2005;7: $153-162$.

23. Tsuchida $\mathrm{T}$, Okazawa $\mathrm{H}$, Mori $\mathrm{T}$, et al. In vivo imaging of estrogen receptor concentration in the endometrium and myometrium using FES PET: influence of menstrual cycle and endogenous estrogen level. Nucl Med Biol. 2007;34: 205-210.

24. Dehdashti F, Mortimer JE, Trinkaus K, et al. PET-based estradiol challenge as a predictive biomarker of response to endocrine therapy in women with estrogen-receptor-positive breast cancer. Breast Cancer Res Treat. 2009;113: 509-517.

25. Linden HM, Stekhova SA, Link JM, et al. Quantitative fluoroestradiol positron emission tomography imaging predicts response to endocrine treatment in breast cancer. J Clin Oncol. 2006;24:2793-2799.

26. Shokeen M, Anderson CJ. Molecular imaging of cancer with copper-64 radiopharmaceuticals and positron emission tomography (PET). Acc Chem Res. 2009;42:832-841.

27. Mamat C, Ramenda T, Wuest FR. Recent application of click chemistry for the synthesis of radiotracers for molecular imaging. Mini Rev Org Chem. 2009;6:21-34.

28. Elizarov AM. Microreactors for radiopharmaceutical synthesis. Lab Chip. 2009;9: 1326-1333.

29. Schottelius M, Wester H-J. Molecular imaging targeting peptide receptors. Methods. 2009;48:161-177.

30. Dunphy MPS, Lewis JS. Radiopharmaceuticals in preclinical and clinical development for monitoring of therapy with PET. J Nucl Med. 2009;50 (suppl 1):106S-121S.

31. Manning HC, Lander A, McKinley E, Mutic NJ. Accelerating the development of novel molecular imaging probes: a role for high-throughput screening. J Nucl Med. 2008;49:1401-1404.

32. Wahlberg E, Lendel C, Helgstrand M, et al. Affibody in complex with a target protein: structure and coupled folding. Proc Natl Acad Sci USA. 2003;100:3185-3190.

33. Kramer-Marek G, Kiesewetter DO, Martiniova L, Jagoda E, Lee SB, Capala J. $\left[{ }^{18}\right.$ F]FBEM-ZHER2:342-Affibody molecule: a new molecular tracer for in vivo monitoring of HER2 expression by positron emission tomography. Eur J Nucl Med Mol Imaging. 2008;35:1008-1018.

34. Jennings LE, Long NJ. "Two is better than one": probes for dual modality molecular imaging. Chem Commun (Camb). 2009;24:3511-3524.

35. Cheon J, Lee J-H. Synergistically integrated nanoparticles as multimodal probes for nanobiotechnology. Acc Chem Res. 2008;41:1630-1640.

36. Cherry SR, Louie AY, Jacobs RE. The integration of positron emission tomography with magnetic resonance imaging. Proc IEEE. 2008;96:416438.

37. Lee HY, Li Z, Chen K, et al. PET/MRI dual modality tumor imaging using arginine-glycine-aspartic (RGD)-conjugated radiolabeled iron oxide nanoparticles. J Nucl Med. 2008;49:1371-1379.

38. Sun G, Hagooly A, Xu J, et al. Facile, efficient approach to accomplish tunable chemistries and variable biodistributions for shell cross-linked nanoparticles. Biomacromolecules. 2008;9:1997-2006.

39. Bentolila LA, Ebenstein Y, Weiss S. Quantum dots for in vivo small-animal imaging. J Nucl Med. 2009;50:493-496.

40. Michalet X, Pinaud FF, Bentolila LA, et al. Quantum dots for lice cells, in vivo imaging and diagnostics. Science. 2005;307:538-544.

41. Bhushan KR, Misra P, Liu F, Mathur S, Lenkinski RE, Frangioni JV. Detection of breast cancer microcalcifications using a dual-modality SPECT/NIR fluorescent probe. J Am Chem Soc. 2008;130:17648-17649.

42. Hynynen K. Macromolecular delivery across the blood-brain barrier. In: Belting M. Macromolecular Drug Delivery Methods and Protocols: Methods in Molecular Biology. Vol 480. Totowa, NJ: Humana Press; 2009:1-11.
43. Willmann JK, Cheng Z, Davis C, et al. Targeted microbubbles for imaging tumor angiogenesis: assessment of whole-body biodistribution with dynamic microPET in mice. Radiology. 2008;249:212-219.

44. Lazaraova N, Causey P, Foster FS, Harasiewicz K, Manouchehhri S, Valliant J. Ultrasound/SPECT dual-modality imaging platform using ${ }^{99 \mathrm{~m}} \mathrm{Tc}$-labeled microbubbles [abstract]. J Nucl Med. 2009;50(suppl 2):149P.

45. Boerman OC, Oyen WJG. Multimodality probes: amphibian cars for molecular imaging. J Nucl Med. 2008;49:1213-1214.

46. Frangioni JV. New technologies for human cancer imaging. J Clin Oncol. 2008;26:4012-4021.

47. Raylman RR, Majewski S, Smith MF, et al. The positron emission mammography/tomography breast imaging and biopsy system (PEM/PET): design, construction and phantom-based measurements. Phys Med Biol. 2008;53: 637-653.

48. Duarte GM, Cabello C, Torresan RZ, et al. Radioguided intraoperative margins evaluation (RIME): preliminary results of a new technique to aid breast cancer resection. Eur J Surg Oncol. 2007;33:1150-1157.

49. Allweis TM, Kaufman Z, Lelcuk S, et al. A prospective randomized, controlled, multicenter study of a real-time intraoperative probe for positive margin detection in breast conserving surgery. Am J Surg. 2008;196:483489.

50. Iagaru A, Peterson D, Quon A, et al. ${ }^{123}$ I MIBG mapping with intraoperative gamma probe for recurrent neuroblastoma. Mol Imaging Biol. 2008;10:1923.

51. Strong VE, Humm J, Russo P, et al. A novel method to localize antibody-targeted cancer deposits intraoperatively using handheld PET beta and gamma probes. Surg Endosc. 2008;22:386-391.

52. Rostovtsev VV, Green LG, Fokin VV, Sharpless KB. A stepwise huisgen cycloaddition process: copper(I)-catalyzed regioselective "ligation" of azides and terminal alkynes. Angew Chem Int Ed Engl. 2002;41:2596-2599.

53. Tornoe CW, Christensen C, Meldal M. Peptidotriazoles on solid phase: [1,2,3]-triazoles by regiospecific copper(I)-catalyzed 1,3-dipolar cycloadditions of terminal alkynes to azides. J Org Chem. 2002;67:3057-3064.

54. Marik J, Sutcliffe JL. Click for PET: rapid preparation of $\left[{ }^{18} \mathrm{~F}\right]$ fluoropeptides using $\mathrm{Cu}^{\mathrm{I}}$ catalyzed 1,3-dipolar cycloaddition. Tetrahedron Lett. 2006;47:66816684 .

55. Sirion U, Kim HJ, Lee JH, et al. efficient F-18 labeling method for PET study: huisgen 1,3-dipolar cycloaddition of bioactive substances and F-18 labeled compounds. Tetrahedron Lett. 2007;48:3953-3957.

56. Knör S, Modlinger A, Poethko T, Schottelius M, Wester H-J, Kessler H. Synthesis of novel 1,4,7,10-tetraazacyclodecane-1,4,7,10-tetraacetic acid (DOTA) derivatives for chemoselective attachment to unprotected polyfunctionalized compounds. Chemistry. 2007;13:6082-6090.

57. Glaser M, Årstad E. "Click labeling" with 2-[ $\left[{ }^{18} \mathrm{~F}\right]$ fluoroethylazide for positron emission tomography. Bioconjug Chem. 2007;18:989-993.

58. Glaser M, Årstad E, Luthra SK, Robins EG. Two-step radiosynthesis of $\left[{ }^{18} \mathrm{~F}\right] \mathrm{N}-$ succinimidyl-4-fluorobenzoate ([ $\left.\left.{ }^{18} \mathrm{~F}\right] \mathrm{SFB}\right)$. J Labelled Comp Radiopharm. 2009;52: 327-330.

59. Mindt TL, Müller C, Stuker F, et al. A "click chemistry" approach to the efficient synthesis of multiple imaging probes derived from a single precursor. Bioconjug Chem. 2009;20:1940-1949.

60. Mindt TL, Schweinsberg C, Brans L, et al. A click approach to structurally diverse conjugates containing a central di-1,2,3-triazole metal chelate. Chem Med Chem. 2009;4:529-539.

61. Ross TL, Honer M, Lam PYH, et al. Fluorine-18 click radiosynthesis and preclinical evaluation of a new ${ }^{18} \mathrm{~F}$-labeled folic acid derivative. Bioconjug Chem. 2008;19:2462-2470.

62. Struthers H, Spingler B, Mindt TL, Schibli R. Click-to-chelate: design and incorporation of triazole-containing metal-chelating systems into biomolecules of diagnostic and therapeutic interest. Chemistry. 2008;14: 6173-6183.

63. Stephenson KA, Banerjee SR, Besanger T, et al. Bridging the gap between in vitro and in vivo imaging: isostructural $\mathrm{Re}$ and ${ }^{99 \mathrm{~m}} \mathrm{Tc}$ complexes for correlating fluorescence and radioimaging studies. J Am Chem Soc. 2004;126:85988599.

64. Stephenson KA, Reid LC, Zubieta J, et al. Synthesis and screening of a library of $\mathrm{Re} / \mathrm{Tc}$-based amyloid probes derived from $\beta$-breaker peptides. Bioconjug Chem. 2008;19:1087-1094.

65. Schaffer P, Gleave JA, Lemon JA, et al. Isostructural fluorescent and radioactive probes for monitoring neural stem and progenitor cell transplants. Nucl Med Biol. 2008;35:159-169.

66. Hausner SH, Kukis DL, Gagnon MKJ, et al. Evaluation of $\left[{ }^{64} \mathrm{Cu}\right] \mathrm{Cu}-\mathrm{DOTA}$ and $\left[{ }^{64} \mathrm{Cu}\right] \mathrm{Cu}-\mathrm{CBTE} 2 \mathrm{~A}$ chelates for targeted positron emission tomography with alpha $_{\mathrm{v}}$ beta $_{6}$-specific peptide. Mol Imaging. 2009;8:111-121. 
67. Parrott MC, Benhabbour SR, Saab C, et al. Synthesis, radiolabeling and bioimaging of high generation polyester dendrimers. J Am Chem Soc. 2009;131:29062916.

68. Almutairi A, Rossin R, Shokeen M, et al. Biodegradable dendritic positronemitting nanoprobes for the noninvasive imaging of angiogenesis. Proc Natl Acad Sci USA. 2009;106:685-690.

69. Hunter DH, Zhu X. Polymer-supported radiopharmaceuticals: $\left[{ }^{131} \mathrm{I}\right] \mathrm{MIBG}$ and [123I]MIBG. J Labelled Comp Radiopharm. 1999;42:653-661.

70. Donovan A, Forbes J, Dorff P, Schaffer P, Babich J, Valliant JF. A new strategy for preparing molecular imaging and therapy agents using fluorine-rich (fluorous) soluble supports. J Am Chem Soc. 2006;128: 3536-3537.

71. McIntee JW, Sundararajan C, Donovan AC, Kovacs MS, Capretta A, Valliant JF. A convenient method for the preparation of fluorous tin derivatives for the fluorous labeling strategy. J Org Chem. 2008;73:8236-8243.

72. Donovan AC, Valliant JF. A convenient solution-phase method for the preparation of meta-iodobenzylguanidine in high effective specific activity. Nucl Med Biol. 2008;35:741-746.

73. Bejot R, Fowler T, Carroll L, et al. Fluorous synthesis of ${ }^{18} \mathrm{f}$ radiotracers with the $\left[{ }^{18} \mathrm{~F}\right]$ fluoride ion: nucleophilic fluorination as the detagging process. Angew Chem Int Ed Engl. 2009;48:586-589.
74. Nekolla SG, Reder S, Saraste A, et al. Evaluation of the novel myocardial perfusion positron-emission tomography tracer ${ }^{18} \mathrm{~F}-\mathrm{BMS}-747158-02$ : comparison to ${ }^{13} \mathrm{~N}$-ammonia and validation with microspheres in a pig model. Circulation. 2009;119:2333-2342.

75. Marshall RC, Powers-Risius P, Reutter BW, et al. Kinetic analysis of ${ }^{18} \mathrm{~F}-$ fluorodihydrorotenone as a deposited myocardial flow tracer: comparison to ${ }^{201}$ Tl. J Nucl Med. 2004;45:1950-1959.

76. Liu S, Kim Y-S, Zhai S, Shi J, Hou G. Evaluation of ${ }^{64} \mathrm{Cu}(\mathrm{DO} 3 \mathrm{~A}-\mathrm{xy}-\mathrm{TPEP})$ as a potential radiotracer for monitoring tumor multidrug resistance. Bioconjug Chem. 2009;20:790-798.

77. Plössl K, Chandra R, Qu W, et al. A novel gallium bisaminothiolate complex as a myocardial perfusion imaging agent. Nucl Med Biol. 2008;35:83-90.

78. Fani M, Andre JP, Maecke HR. ${ }^{68}$ Ga-PET: a powerful generator-based alternative to cyclotron-based PET radiopharmaceuticals. Contrast Media Mol Imaging. 2008;3:67-77.

79. Hruska CB, Phillips SW, Whaley DH, Rhodes DJ, O'Connor MK. Molecular breast imaging: use of a dual-head dedicated gamma camera to detect small breast tumors. AJR. 2008;191:1805-1815.

80. Hruska CB, Boughey JC, Phillips SW, et al. Scientific Impact Recognition Award: molecular breast imaging: a review of the Mayo Clinic experience. Am J Surg. 2008;196:470-476. 